\title{
Strong Convergence Theorems for an Infinite Family of Equilibrium Problems and Fixed Point Problems for an Infinite Family of Asymptotically Strict Pseudocontractions
}

\author{
Shenghua Wang, ${ }^{1}$ Shin Min Kang, ${ }^{2}$ and Young Chel Kwun ${ }^{3}$ \\ ${ }^{1}$ School of Applied Mathematics and Physics, North China Electric Power University, \\ Baoding 071003, China \\ ${ }^{2}$ Department of Mathematics and RINS, Gyeongsang National University, Jinju 660-701, Republic of Korea \\ ${ }^{3}$ Department of Mathematics, Dong-A University, Pusan 614-714, Republic of Korea
}

Correspondence should be addressed to Young Chel Kwun, yckwun@dau.ac.kr

Received 12 October 2010; Accepted 29 January 2011

Academic Editor: Jong Kim

Copyright ( $) 2011$ Shenghua Wang et al. This is an open access article distributed under the Creative Commons Attribution License, which permits unrestricted use, distribution, and reproduction in any medium, provided the original work is properly cited.

We prove a strong convergence theorem for an infinite family of asymptotically strict pseudocontractions and an infinite family of equilibrium problems in a Hilbert space. Our proof is simple and different from those of others, and the main results extend and improve those of many others.

\section{Introduction}

Let $C$ be a closed convex subset of a Hilbert space $H$. Let $S: C \rightarrow H$ be a mapping and if there exists an element $x \in C$ such that $x=S x$, then $x$ is called a fixed point of $S$. The set of fixed points of $S$ is denoted by $F(S)$. Recall that

(1) $S$ is called nonexpansive if

$$
\|S x-S y\| \leq\|x-y\|, \quad \forall x, y \in C
$$

(2) $S$ is called asymptotically nonexpansive [1] if there exists a sequence $\left\{k_{n}\right\} \subset[1, \infty)$ with $k_{n} \rightarrow 1$ such that 


$$
\left\|S^{n} x-S^{n} y\right\| \leq k_{n}\|x-y\|, \quad \forall x, y \in C, n \geq 1
$$

(3) $S$ is called to be a $\kappa$-strict pseudo-contraction [2] if there exists a constant $\mathcal{\kappa}$ with $0 \leq \kappa<1$ such that

$$
\|S x-S y\|^{2} \leq\|x-y\|^{2}+\kappa\|(x-y)-(S x-S y)\|^{2}, \quad \forall x, y \in C,
$$

(4) $S$ is called an asymptotically $\kappa$-strict pseudo-contraction $[3,4]$ if there exists a constant $\kappa$ with $0 \leq \kappa<1$ and a sequence $\left\{\gamma_{n}\right\} \subset[0, \infty)$ with $\lim _{n \rightarrow \infty} \gamma_{n}=0$ such that

$$
\left\|S^{n} x-S^{n} y\right\|^{2} \leq\left(1+\gamma_{n}\right)\|x-y\|^{2}+\kappa\left\|(x-y)-\left(S^{n} x-S^{n} y\right)\right\|^{2}, \quad \forall x, y \in C, n \geq 1 .
$$

It is clear that every asymptotically nonexpansive mapping is an asymptotically 0strict pseudo-contraction and every $\kappa$-strict pseudo-contraction is an asymptotically $\kappa$-strict pseudo-contraction with $\gamma_{n}=0$ for all $n \geq 1$. Moreover, every asymptotically $\kappa$-strict pseudo-contraction with sequence $\left\{\gamma_{n}\right\}$ is uniformly $L$-Lispchitzian, where $L=\sup \{(\kappa+$ $\left.\left.\sqrt{1+\gamma_{n}(1-\kappa)}\right) /(1-\kappa): n \geq 1\right\}$ and the fixed point set of asymptotically $\kappa$-strict pseudocontraction is closed and convex; see [3, Proposition 2.6].

Let $\Phi$ be a bifunction from $C \times C$ to $\mathbb{R}$, where $\mathbb{R}$ is the set of real numbers. The equilibrium problem for $\Phi: C \times C \rightarrow \mathbb{R}$ is to find $x \in C$ such that $\Phi(x, y) \geq 0$ for all $y \in C$. The set of such solutions is denoted by $\mathrm{EP}(\Phi)$.

In 2007, S. Takahashi and W. Takahashi [5] first introduced an iterative scheme by the viscosity approximation method for finding a common element of the set of solutions of the equilibrium problem and the set of fixed points of a nonexpansive mapping in a Hilbert space $H$ and proved a strong convergence theorem which is connected with Combettes and Hirstoaga's result [6] and Wittmann's result [7]. More precisely, they gave the following theorem.

Theorem 1.1 (see [5]). Let $C$ be a nonempty closed convex subset of $H$. Let $\Phi$ be a bifunction from $C \times C$ to $\mathbb{R}$ satisfying the following assumptions:

(A1) $\Phi(x, x)=0$ for all $x \in C$;

(A2) $\Phi$ is monotone, that is, $\Phi(x, y)+\Phi(y, x) \leq 0$ for all $x, y \in C$;

(A3) for all $x, y, z \in C$,

$$
\lim _{t \downarrow 0} \Phi(t z+(1-t) x, y) \leq \Phi(x, y)
$$

(A4) for all $x \in C, y \mapsto \Phi(x, y)$ is convex and lower semicontinuous. 
Fixed Point Theory and Applications

Let $S: C \rightarrow H$ be a nonexpansive mapping such that $F(S) \cap \mathrm{EP}(\Phi) \neq \emptyset, f: H \rightarrow H$ be a contraction and $\left\{x_{n}\right\},\left\{u_{n}\right\}$ be the sequences generated by

$$
\begin{gathered}
x_{1} \in H, \\
\Phi\left(u_{n}, y\right)+\frac{1}{r_{n}}\left\langle y-u_{n}, u_{n}-x_{n}\right\rangle \geq 0, \quad \forall y \in C, \\
x_{n+1}=\alpha_{n} f\left(x_{n}\right)+\left(1-\alpha_{n}\right) S u_{n}, \quad \forall n \geq 1,
\end{gathered}
$$

where $\left\{\alpha_{n}\right\} \subset[0,1]$ and $\left\{r_{n}\right\} \subset(0, \infty)$ satisfy the following conditions:

$$
\begin{gathered}
\lim _{n \rightarrow \infty} \alpha_{n}=0, \quad \sum_{n=1}^{\infty} \alpha_{n}=\infty, \quad \sum_{n=1}^{\infty}\left|\alpha_{n+1}-\alpha_{n}\right|<\infty, \\
\liminf _{n \rightarrow \infty} r_{n}>0, \quad \sum_{n=1}^{\infty}\left|r_{n+1}-r_{n}\right|<\infty
\end{gathered}
$$

Then, the sequences $\left\{x_{n}\right\}$ and $\left\{u_{n}\right\}$ converge strongly to $z \in F(S) \cap \operatorname{EP}(\Phi)$, where $z=$ $P_{F(S) \cap E P(\Phi)} f(z)$.

In [8], Tada and Takahashi proposed a hybrid algorithm to find a common element of the set of fixed points of a nonexpansive mapping and the set of solutions of an equilibrium problem and proved the following strong convergence theorem.

Theorem 1.2 (see [8]). Let $C$ be a nonempty closed convex subset of a Hilbert space $H$. Let $\Phi$ be a bifunction from $C \times C \rightarrow \mathbb{R}$ satisfying (A1)-(A4) and let $S$ be a nonexpansive mapping of $C$ into $H$ such that $F(S) \cap \operatorname{EP}(\Phi) \neq \emptyset$. Let $\left\{x_{n}\right\}$ and $\left\{u_{n}\right\}$ be sequences generated by $x_{1}=x \in H$ and

$$
\begin{gathered}
u_{n} \in C \text { such that } \Phi\left(u_{n}, y\right)+\frac{1}{r_{n}}\left\langle y-u_{n}, u_{n}-x_{n}\right\rangle \geq 0, \quad \forall y \in C, \\
w_{n}=\left(1-\alpha_{n}\right) x_{n}+\alpha_{n} S u_{n}, \\
C_{n}=\left\{z \in H:\left\|w_{n}-z\right\| \leq\left\|x_{n}-z\right\|\right\}, \\
D_{n}=\left\{z \in H:\left\langle x_{n}-z, x-x_{n}\right\rangle \geq 0\right\}, \\
x_{n+1}=P_{C_{n} \cap D_{n}} x, \quad \forall n \geq 1,
\end{gathered}
$$

where $\left\{\alpha_{n}\right\} \subset[a, 1]$ for some $a \in(0,1)$ and $\left\{r_{n}\right\} \subset(0, \infty)$ satisfies $\liminf _{n \rightarrow \infty} r_{n}>0$. Then $\left\{x_{n}\right\}$ converges strongly to $P_{F(S) \cap E P(\Phi)} x$.

Many methods have been proposed to solve the equilibrium problems and fixed point problems; see [9-13].

Recently, Kim and $\mathrm{Xu}$ [3] proposed a hybrid algorithm for finding a fixed point of an asymptotically $\kappa$-strict pseudo-contraction and proved a strong convergence theorem in a Hilbert space. 
Theorem 1.3 (see [3]). Let $C$ be a closed convex subset of a Hilbert space $H$. Let $T: C \rightarrow C$ be an asymptotically $\kappa$-strict pseudo-contraction for some $0 \leq \kappa<1$. Assume that $F(T)$ is nonempty and bounded. Let $\left\{x_{n}\right\}$ be the sequence generated by the following algorithm:

$$
\begin{gathered}
x_{0} \in C \text { chosen arbitrarily, } \\
y_{n}=\alpha_{n} x_{n}+\left(1-\alpha_{n}\right) T^{n} x_{n}, \\
C_{n}=\left\{z \in H:\left\|y_{n}-z\right\| \leq\left\|x_{n}-z\right\|^{2}+\left[\kappa-\alpha_{n}\left(1-\alpha_{n}\right)\right]\left\|x_{n}-T^{n} x_{n}\right\|^{2}+\theta_{n}\right\}, \\
D_{n}=\left\{z \in H:\left\langle x_{n}-z, x_{0}-x_{n}\right\rangle \geq 0\right\}, \\
x_{n+1}=P_{C_{n} \cap D_{n}} x_{0}, \quad \forall n \geq 1,
\end{gathered}
$$

where

$$
\theta_{n}=\Delta_{n}^{2}\left(1-\alpha_{n}\right) \gamma_{n} \longrightarrow 0 \quad(n \longrightarrow \infty), \quad \Delta_{n}=\sup \left\{\left\|x_{n}-z\right\|: z \in F(T)\right\}<\infty
$$

Assume that the control sequence $\left\{\alpha_{n}\right\}$ is chosen such that $\limsup _{n \rightarrow \infty} \alpha_{n}<1-\kappa$. Then $\left\{x_{n}\right\}$ converges strongly to $P_{F(T)} x_{0}$.

In this paper, motivated by [3, 8], we propose a new algorithm for finding a common element of the set of fixed points of an infinite family of asymptotically strict pseudocontractions and the set of solutions of an infinite family of equilibrium problems and prove a strong convergence theorem. Our proof is simple and different from those of others, and the main results extend and improve those Kim and $\mathrm{Xu}$ [3], Tada and Takahashi [8], and many others.

\section{Preliminaries}

Let $H$ be a Hilbert space, and let $C$ be a nonempty closed convex subset of $H$. It is well known that, for all $x, y \in C$ and $t \in[0,1]$,

$$
\|t x+(1-t) y\|^{2}=t\|x\|^{2}+(1-t)\|y\|^{2}-t(1-t)\|x-y\|,
$$

and hence

$$
\|t x+(1-t) y\|^{2} \leq t\|x\|^{2}+(1-t)\|y\|^{2},
$$

which implies that

$$
\left\|\sum_{i=1}^{n} t_{i} x_{i}\right\|^{2} \leq \sum_{i=1}^{n} t_{i}\left\|x_{i}\right\|^{2}
$$

for all $\left\{x_{i}\right\} \subset H$ and $\left\{t_{i}\right\} \subset[0,1]$ with $\sum_{i=1}^{n} t_{i}=1$. 
For any $x \in H$, there exists a unique nearest point in $C$, denoted by $P_{C} x$, such that

$$
z=P_{C} x \Longleftrightarrow\langle x-z, z-y\rangle \geq 0, \quad \forall y \in C
$$

Let $I$ denote the identity operator of $H$, and let $\left\{x_{n}\right\}$ be a sequence in a Hilbert space $H$ and $x \in H$. Throughout the rest of the paper, $x_{n} \rightarrow x$ denotes the strong convergence of $\left\{x_{n}\right\}$ to $x$.

We need the following lemmas for our main results in this paper.

Lemma 2.1 (see [14]). Let $C$ be a nonempty closed convex subset of a Hilbert space $H$. Let $\Phi$ be a bifunction from $C \times C$ to $\mathbb{R}$ satisfying (A1)-(A4). Let $r>0$ and $x \in H$. Then there exists $z \in C$ such that

$$
\Phi(z, y)+\frac{1}{r}\langle y-z, z-x\rangle \geq 0, \quad \forall y \in C
$$

Lemma 2.2 (see [6]). Let $C$ be a nonempty closed convex subset of a Hilbert space $H$. Let $\Phi$ be a bifunction from $C \times C$ to $\mathbb{R}$ satisfying (A1)-(A4). For any $r>0$ and $x \in H$, define a mapping $T_{r}: H \rightarrow C$ as follows:

$$
T_{r} x=\left\{z \in C: \Phi(z, y)+\frac{1}{r}\langle y-z, z-x\rangle \geq 0, \forall y \in C\right\}, \quad \forall x \in H
$$

Then the following hold:

(1) $T_{r}$ is single-valued,

(2) $T_{r}$ is firmly nonexpansive, that is, for any $x, y \in H$,

$$
\left\|T_{r} x-T_{r} y\right\|^{2} \leq\left\langle T_{r} x-T_{r} y, x-y\right\rangle
$$

(3) $F\left(T_{r}\right)=\mathrm{EP}(\Phi)$, and

(4) $\mathrm{EP}(\Phi)$ is closed and convex.

\section{Main Results}

Now, we are ready to give our main results.

Lemma 3.1. Let $C$ be a nonempty closed convex subset of a Hilbert space $H$. Let $T: C \rightarrow C$ be an asymptotically $\kappa$-strict pseudo-contraction with sequence $\left\{\gamma_{n}\right\} \subset[0, \infty)$ such that $F(T) \neq \emptyset$. Assume that $\left\{\beta_{n}\right\} \subset[\kappa, 1]$ and define a mapping $S_{n}=\beta_{n} I+\left(1-\beta_{n}\right) T^{n}$ for each $n \geq 1$. Then the following hold:

$$
\begin{gathered}
\left\|S_{n} x-S_{n} y\right\|^{2} \leq\left(1+\gamma_{n}\right)\|x-y\|^{2}, \quad \forall x, y \in C, \\
\left\|S_{n} x-x\right\|^{2} \leq \gamma_{n}\left\|x-x^{*}\right\|^{2}+2\left\langle x-S_{n} x, x-x^{*}\right\rangle, \quad \forall x \in C, x^{*} \in F(T) .
\end{gathered}
$$


Proof. For all $x, y \in C$, we have

$$
\begin{aligned}
\left\|S_{n} x-S_{n} y\right\|^{2}= & \left\|\beta_{n}(x-y)+\left(1-\beta_{n}\right)\left(T^{n} x-T^{n} y\right)\right\|^{2} \\
= & \beta_{n}\|x-y\|^{2}+\left(1-\beta_{n}\right)\left\|T^{n} x-T^{n} y\right\|^{2}-\beta_{n}\left(1-\beta_{n}\right)\left\|\left(I-T^{n}\right) x-\left(I-T^{n}\right) y\right\|^{2} \\
\leq & \beta_{n}\|x-y\|^{2}+\left(1-\beta_{n}\right)\left[\left(1+\gamma_{n}\right)\|x-y\|^{2}+\kappa\left\|\left(I-T^{n}\right) x-\left(I-T^{n}\right) y\right\|^{2}\right] \\
& -\beta_{n}\left(1-\beta_{n}\right)\left\|\left(I-T^{n}\right) x-\left(I-T^{n}\right) y\right\|^{2} \\
= & \beta_{n}\|x-y\|^{2}+\left(1-\beta_{n}\right)\left(1+\gamma_{n}\right)\|x-y\|^{2} \\
& +\left(1-\beta_{n}\right)\left(\kappa-\beta_{n}\right)\left\|\left(I-T^{n}\right) x-\left(I-T^{n}\right) y\right\|^{2} \\
\leq & \beta_{n}\|x-y\|^{2}+\left(1-\beta_{n}\right)\left(1+\gamma_{n}\right)\|x-y\|^{2} \\
\leq & \left(1+\gamma_{n}\right)\|x-y\|^{2} .
\end{aligned}
$$

By this result, for all $x \in C$ and $x^{*} \in F(T)$, we have

$$
\begin{aligned}
\left(1+\gamma_{n}\right)\left\|x-x^{*}\right\|^{2} & \geq\left\|S_{n} x-S_{n} x^{*}\right\|^{2}=\left\|S_{n} x-x+x-x^{*}\right\|^{2} \\
& =\left\|S_{n} x-x\right\|^{2}+\left\|x-x^{*}\right\|^{2}+2\left\langle S_{n} x-x, x-x^{*}\right\rangle,
\end{aligned}
$$

and hence

$$
\left\|S_{n} x-x\right\|^{2} \leq \gamma_{n}\left\|x-x^{*}\right\|^{2}+2\left\langle x-S_{n} x, x-x^{*}\right\rangle .
$$

This completes the proof.

Lemma 3.2. Let $C$ be a nonempty closed subset of a Hilbert space $H$. Let $T: C \rightarrow C$ be an asymptotically $\kappa$-strict pseudo-contraction with sequence $\left\{\gamma_{n}\right\} \subset[0, \infty)$ satisfying $\gamma_{n} \rightarrow 0$ as $n \rightarrow \infty$. Let $\left\{z_{n}\right\}$ be a sequence in $C$ such that $\left\|z_{n}-z_{n+1}\right\| \rightarrow 0$ and $\left\|z_{n}-T^{n} z_{n}\right\| \rightarrow 0$ as $n \rightarrow \infty$. Then $\left\|z_{n}-T z_{n}\right\| \rightarrow 0$ as $n \rightarrow \infty$.

Proof. The proof method of this lemma is mainly from [15, Lemma 2.7]. Since $T$ is an asymptotically $\kappa$-strict pseudo-contraction, we obtain from [3, Proposition 2.6] that

$$
\left\|T^{n+1} z_{n}-T^{n+1} z_{n+1}\right\| \leq L\left\|z_{n}-z_{n+1}\right\|
$$

where $L=\sup \left\{\left(\kappa+\sqrt{1+\gamma_{n}(1-\kappa)}\right) /(1-\kappa): n \geq 1\right\}$. Note that $\left\|z_{n}-z_{n+1}\right\| \rightarrow 0$, which implies that $\left\|T^{n+1} z_{n}-T^{n+1} z_{n+1}\right\| \rightarrow 0$, and observe that

$$
\begin{aligned}
\left\|z_{n}-T z_{n}\right\| & \leq\left\|z_{n}-z_{n+1}\right\|+\left\|z_{n+1}-T^{n+1} z_{n+1}\right\|+\left\|T^{n+1} z_{n+1}-T^{n+1} z_{n}\right\|+\left\|T^{n+1} z_{n}-T z_{n}\right\| \\
& \leq(1+L)\left\|z_{n}-z_{n+1}\right\|+\left\|z_{n+1}-T^{n+1} z_{n+1}\right\|+\left\|T^{n+1} z_{n}-T z_{n}\right\| .
\end{aligned}
$$


Since $T$ is uniformly Lipschitzian, $T$ is uniformly continuous. So we have

$$
\left\|T^{n+1} z_{n}-T z_{n}\right\| \longrightarrow 0 \quad \text { as } n \longrightarrow \infty
$$

It follows from $\left\|z_{n}-z_{n+1}\right\| \rightarrow 0$ and $\left\|z_{n}-T^{n} z_{n}\right\| \rightarrow 0$ as $n \rightarrow \infty$ that $\lim _{n \rightarrow \infty}\left\|z_{n}-T z_{n}\right\|=0$. This completes the proof.

Let $H$ be a Hilbert space, and, let $C$ be a nonempty closed and convex subset of $H$. Let $\left\{\Phi_{n}\right\}$ be a countable family of bifunctions from $C \times C$ to $\mathbb{R}$ satisfying (A1)-(A4) and let $\left\{r_{n}\right\}$ be a real number sequence in $(r, \infty)$ with $r>0$. Define

$$
T_{r_{i}} x=\left\{z \in C: \Phi_{i}(z, y)+\frac{1}{r_{i}}\langle y-z, z-x\rangle \geq 0, \forall y \in C\right\}, \quad \forall x \in H .
$$

Lemma 2.2 shows that every $T_{r_{i}}(i \geq 1)$ is a firmly nonexpansive mapping and hence nonexpansive and $F\left(T_{r_{i}}\right)=\operatorname{EP}\left(\Phi_{i}\right)$.

Theorem 3.3. Let $C$ be a nonempty closed convex subset of a Hilbert space $H$. Let $\left\{T_{i}\right\}: C \rightarrow C$ be an infinite family of asymptotically $\kappa_{i}$-strict pseudocontractions with the sequence $\left\{\gamma_{i, n}\right\} \subset[0, \infty)$ satisfying $\gamma_{i, n} \rightarrow 0$ as $n \rightarrow \infty$ for each $i \geq 1$ and $\gamma_{1, n} \geq \gamma_{i, n}$ for each $i \geq 1$ and $n \geq 1$. Let $\left\{\Phi_{n}\right\}$ be a countable family of bifunctions from $C \times C$ to $\mathbb{R}$ satisfying (A1)-(A4). Assume that $\Omega=$ $\bigcap_{i=1}^{\infty}\left(F\left(T_{i}\right) \cap \mathrm{EP}\left(\Phi_{i}\right)\right)$ is nonempty and bounded. Set $\alpha_{0}=1$ and $\theta_{0}=1$. Assume that $\left\{\alpha_{i}\right\}$ is a strictly decreasing sequence in $[0, a]$ for some $0<a<1,\left\{\theta_{n}\right\}$ is a strictly decreasing sequence in $(0,1),\left\{\beta_{i, n}\right\}$ is a sequence in $\left[\kappa_{i}, \kappa\right)$ with $0<\kappa_{i}<\kappa<1$ for each $i \geq 1$, and $\left\{r_{n}\right\}$ is a sequence in $(r, \infty)$ with $r>0$. The sequence $\left\{x_{n}\right\}$ is generated by $x_{1}=x \in C$ and

$$
\begin{gathered}
z_{n}=\theta_{n} x_{n}+\sum_{i=1}^{n}\left(\theta_{i-1}-\theta_{i}\right) T_{r_{i}} x_{n}, \\
w_{n}=\alpha_{n} x_{n}+\sum_{i=1}^{n}\left(\alpha_{i-1}-\alpha_{i}\right)\left(\beta_{i, n} I+\left(1-\beta_{i, n}\right) T_{i}^{n}\right) z_{n}, \\
C_{n}=\left\{v \in C:\left\|w_{n}-v\right\| \leq\left\|x_{n}-v\right\|+\lambda_{n}\right\}, \\
D_{n}=\bigcap_{j=1}^{n} C_{j}, \\
x_{n+1}=P_{D_{n}} x, \quad \forall n \geq 1,
\end{gathered}
$$

where $\left\{T_{r_{i}}\right\}$ is defined by (3.8) and

$$
\lambda_{n}=\left(1-\alpha_{n}\right) \gamma_{1, n} \Delta_{n} \longrightarrow 0 \quad(n \longrightarrow \infty), \quad \Delta_{n}=\sup \left\{\left\|x_{n}-v\right\|: v \in \Omega\right\}
$$

Then $\left\{x_{n}\right\}$ converges strongly to $P_{\Omega} x$. 
Proof. We show first that the sequence $\left\{x_{n}\right\}$ is well defined. Obviously, $C_{n}$ is closed for all $n \geq 1$. Since

$$
\left\|w_{n}-v\right\| \leq\left\|x_{n}-v\right\|+\lambda_{n}
$$

is equivalent to

$$
\left\|w_{n}-x_{n}\right\|^{2}+2\left\langle w_{n}-x_{n}, x_{n}-z\right\rangle \leq \lambda_{n}
$$

$C_{n}$ is convex for all $n \geq 1$. So $D_{n}=\bigcap_{j=1}^{n} C_{j}$ is also closed and convex for all $n \geq 1$.

For each $n \geq 1$ and $i \geq 1$, put $S_{i, n}=\beta_{i, n} I+\left(1-\beta_{i, n}\right) T_{i}^{n}$. Let $p \in \Omega$. Note that $\theta_{0}=1,\left\{\theta_{n}\right\}$ is strictly decreasing and each $T_{r_{i}}$ is firmly nonexpansive. Hence we have

$$
\begin{aligned}
\left\|z_{n}-p\right\| & \leq \theta_{n}\left\|x_{n}-p\right\|+\sum_{i=1}^{n}\left(\theta_{i-1}-\theta_{i}\right)\left\|T_{r_{i}} x_{n}-p\right\| \\
& \leq \theta_{n}\left\|x_{n}-p\right\|+\sum_{i=1}^{n}\left(\theta_{i-1}-\theta_{i}\right)\left\|x_{n}-p\right\| \\
& \leq \theta_{n}\left\|x_{n}-p\right\|+\left(1-\theta_{n}\right)\left\|x_{n}-p\right\| \\
& =\left\|x_{n}-p\right\|, \quad \forall n \geq 1 .
\end{aligned}
$$

Since $\alpha_{0}=1$ and $\left\{\alpha_{n}\right\}$ is strictly decreasing, by (3.13) and Lemma 3.1, we have

$$
\begin{aligned}
\left\|w_{n}-p\right\| & \leq \alpha_{n}\left\|x_{n}-p\right\|+\sum_{i=1}^{n}\left(\alpha_{i-1}-\alpha_{i}\right)\left\|S_{i, n} z_{n}-p\right\| \\
& \leq \alpha_{n}\left\|x_{n}-p\right\|+\sum_{i=1}^{n}\left(\alpha_{i-1}-\alpha_{i}\right) \sqrt{1+\gamma_{i, n}}\left\|z_{n}-p\right\| \\
& \leq \alpha_{n}\left\|x_{n}-p\right\|+\sum_{i=1}^{n}\left(\alpha_{i-1}-\alpha_{i}\right)\left(1+\gamma_{1, n}\right)\left\|x_{n}-p\right\| \\
& \leq\left\|x_{n}-p\right\|+\lambda_{n} .
\end{aligned}
$$

So we have $p \in C_{n}$ and hence $p \in D_{n}=\bigcap_{j=1}^{n} C_{j}$ for all $n \geq 1$. This shows that $\Omega \subset D_{n}$ for all $n \geq 1$. This implies that the sequence $\left\{x_{n}\right\}$ is well defined. that

Since $\Omega$ is a nonempty closed convex subset of $H$, there exists a unique $z^{*} \in \Omega$ such

$$
z^{*}=P_{\Omega} x
$$

From $x_{n+1}=P_{D_{n}} x$, we have

$$
\left\|x_{n+1}-x\right\| \leq\|z-x\|, \quad \forall z \in D_{n} .
$$


Fixed Point Theory and Applications

Since $z^{*} \in \Omega \subset D_{n}$, we have

$$
\left\|x_{n+1}-x\right\| \leq\left\|z^{*}-x\right\|, \quad \forall n \geq 1
$$

Therefore, $\left\{x_{n}\right\}$ is bounded. From (3.13) and (3.14), $\left\{z_{n}\right\}$ and $\left\{w_{n}\right\}$ are also bounded.

From $x_{n+1}=P_{D_{n}} x$ and $D_{n+1} \subset D_{n}$, one sees that $x_{n+2}=P_{D_{n+1}} x \in D_{n+1} \subset D_{n}$ for all $n \geq 1$. It follows that

$$
\left\|x_{n+1}-x\right\| \leq\left\|x_{n+2}-x\right\|, \quad \forall n \geq 1
$$

Since $\left\{x_{n}\right\}$ is bounded, the sequence $\left\{\left\|x-x_{n}\right\|\right\}$ is bounded and nondecreasing. So there exists $c \in \mathbb{R}$ such that

$$
c=\lim _{n \rightarrow \infty}\left\|x-x_{n}\right\|
$$

Since $x_{n+1}=P_{D_{n}} x \in D_{n}, x_{n+2}=P_{D_{n+1}} x \in D_{n+1} \subset D_{n}$ and $\left(x_{n+1}+x_{n+2}\right) / 2 \in D_{n}$, we have

$$
\begin{aligned}
\left\|x-x_{n+1}\right\|^{2} & \leq\left\|x-\frac{x_{n+1}+x_{n+2}}{2}\right\|^{2} \\
& =\left\|\frac{1}{2}\left(x-x_{n+1}\right)+\frac{1}{2}\left(x-x_{n+2}\right)\right\|^{2} \\
& =\frac{1}{2}\left\|x-x_{n+1}\right\|^{2}+\frac{1}{2}\left\|x-x_{n+2}\right\|^{2}-\frac{1}{4}\left\|x_{n+1}-x_{n+2}\right\|^{2} .
\end{aligned}
$$

So we get

$$
\frac{1}{4}\left\|x_{n+1}-x_{n+2}\right\|^{2} \leq \frac{1}{2}\left\|x-x_{n+2}\right\|^{2}-\frac{1}{2}\left\|x-x_{n+1}\right\|^{2} .
$$

Since $\lim _{n \rightarrow \infty}\left\|x-x_{n+1}\right\|=\lim _{n \rightarrow \infty}\left\|x-x_{n+2}\right\|=c$, we obtain

$$
\lim _{n \rightarrow \infty}\left\|x_{n+1}-x_{n+2}\right\|=0
$$

that is,

$$
\lim _{n \rightarrow \infty}\left\|x_{n}-x_{n+1}\right\|=0
$$

Now, for each $l \geq 1$, from (3.23) we get

$$
\begin{aligned}
\left\|x_{n+l}-x_{n}\right\| & \leq\left\|x_{n+l}-x_{n+l-1}\right\|+\cdots+\left\|x_{n+1}-x_{n}\right\| \\
& \longrightarrow 0 \quad \text { as } n \longrightarrow \infty
\end{aligned}
$$


This implies that there exists an element $\widehat{x} \in C$ such that $x_{n} \rightarrow \widehat{x}$ as $n \rightarrow \infty$.

Next we show that $\hat{x} \in \bigcap_{i=1}^{\infty} F\left(T_{i}\right)$ and $\hat{x} \in \bigcap_{i=1}^{\infty} \operatorname{EP}\left(\Phi_{i}\right)$.

From $x_{n+1} \in C_{n}$, we have

$$
\begin{aligned}
\left\|x_{n}-w_{n}\right\| & \leq\left\|x_{n}-x_{n+1}\right\|+\left\|x_{n+1}-w_{n}\right\| \\
& \leq 2\left\|x_{n}-x_{n+1}\right\|+\lambda_{n} .
\end{aligned}
$$

By (3.10) and (3.23), we obtain

$$
\lim _{n \rightarrow \infty}\left\|x_{n}-w_{n}\right\|=0
$$

For $p \in \Omega$, we have, from Lemma 2.2,

$$
\begin{aligned}
\left\|T_{r_{i}} x_{n}-p\right\|^{2} & =\left\|T_{r_{i}} x_{n}-T_{r_{i}} p\right\|^{2} \\
& \leq\left\langle T_{r_{i}} x_{n}-T_{r_{i}} p, x_{n}-p\right\rangle \\
& =\left\langle T_{r_{i}} x_{n}-p, x_{n}-p\right\rangle \\
& =\frac{1}{2}\left(\left\|T_{r_{i}} x_{n}-p\right\|^{2}+\left\|x_{n}-p\right\|^{2}-\left\|x_{n}-T_{r_{i}} x_{n}\right\|^{2}\right),
\end{aligned}
$$

and hence

$$
\left\|T_{r_{i}} x_{n}-p\right\|^{2} \leq\left\|x_{n}-p\right\|^{2}-\left\|x_{n}-T_{r_{i}} x_{n}\right\|^{2}, \quad \forall i \geq 1 .
$$

Therefore

$$
\begin{aligned}
\left\|z_{n}-p\right\|^{2} & \leq \theta_{n}\left\|x_{n}-p\right\|^{2}+\sum_{i=1}^{n}\left(\theta_{i-1}-\theta_{i}\right)\left\|T_{r_{i}} x_{n}-p\right\|^{2} \\
& \leq \theta_{n}\left\|x_{n}-p\right\|^{2}+\sum_{i=1}^{n}\left(\theta_{i-1}-\theta_{i}\right)\left(\left\|x_{n}-p\right\|^{2}-\left\|x_{n}-T_{r_{i}} x_{n}\right\|^{2}\right) \\
& =\left\|x_{n}-p\right\|^{2}-\sum_{i=1}^{n}\left(\theta_{i-1}-\theta_{i}\right)\left\|x_{n}-T_{r_{i}} x_{n}\right\|^{2} .
\end{aligned}
$$


By (3.29) and Lemma 3.1, we have

$$
\begin{aligned}
\left\|w_{n}-p\right\|^{2} \leq & \alpha_{n}\left\|x_{n}-p\right\|^{2}+\sum_{i=1}^{n}\left(\alpha_{i-1}-\alpha_{i}\right)\left\|S_{i, n} z_{n}-p\right\|^{2} \\
\leq & \alpha_{n}\left\|x_{n}-p\right\|^{2}+\sum_{i=1}^{n}\left(\alpha_{i-1}-\alpha_{i}\right)\left(1+\gamma_{1, n}\right)^{2}\left\|z_{n}-p\right\|^{2} \\
= & \alpha_{n}\left\|x_{n}-p\right\|^{2}+\left(1-\alpha_{n}\right)\left(1+\gamma_{1, n}\right)^{2}\left\|z_{n}-p\right\|^{2} \\
\leq & \alpha_{n}\left\|x_{n}-p\right\|^{2}+\left(1-\alpha_{n}\right)\left(1+\gamma_{1, n}\right)^{2}\left(\left\|x_{n}-p\right\|^{2}-\sum_{i=1}^{n}\left(\theta_{i-1}-\theta_{i}\right)\left\|x_{n}-T_{r_{i}} x_{n}\right\|^{2}\right) \\
= & \left\|x_{n}-p\right\|^{2}+\left(1-\alpha_{n}\right)\left(2 \gamma_{1, n}+\gamma_{1, n}^{2}\right)\left\|x_{n}-p\right\|^{2} \\
& -\left(1-\alpha_{n}\right)\left(1+\gamma_{1, n}\right)^{2} \sum_{i=1}^{n}\left(\theta_{i-1}-\theta_{i}\right)\left\|x_{n}-T_{r_{i}} x_{n}\right\|^{2},
\end{aligned}
$$

and hence

$$
\begin{aligned}
& \left(1-\alpha_{n}\right)\left(1+\gamma_{1, n}\right)^{2} \sum_{i=1}^{n}\left(\theta_{i-1}-\theta_{i}\right)\left\|x_{n}-T_{r_{i}} x_{n}\right\|^{2} \\
& \quad \leq\left\|x_{n}-p\right\|^{2}-\left\|w_{n}-p\right\|^{2}+\left(1-\alpha_{n}\right)\left(2 \gamma_{1, n}+\gamma_{1, n}^{2}\right)\left\|x_{n}-p\right\|^{2} \\
& \quad \leq\left\|x_{n}-w_{n}\right\|\left(\left\|x_{n}-p\right\|+\left\|w_{n}-p\right\|\right)+\left(1-\alpha_{n}\right)\left(2 \gamma_{1, n}+\gamma_{1, n}^{2}\right)\left\|x_{n}-p\right\|^{2} .
\end{aligned}
$$

This shows that

$$
\begin{aligned}
\left(1-\alpha_{n}\right)\left(1+\gamma_{1, n}\right)^{2}\left(\theta_{i-1}-\theta_{i}\right)\left\|x_{n}-T_{r_{i}} x_{n}\right\|^{2} & \\
\leq & \left\|x_{n}-w_{n}\right\|\left(\left\|x_{n}-p\right\|+\left\|w_{n}-p\right\|\right) \\
& +\left(1-\alpha_{n}\right)\left(2 \gamma_{1, n}+\gamma_{1, n}^{2}\right)\left\|x_{n}-p\right\|^{2}, \quad \forall i \geq 1 .
\end{aligned}
$$

Since $\left\{\alpha_{n}\right\} \subset[0, a]$ with $0<a<1, \gamma_{1, n} \rightarrow 0,\left\{\theta_{n}\right\}$ is strictly decreasing and $\left\|x_{n}-w_{n}\right\| \rightarrow 0$, we get

$$
\lim _{n \rightarrow \infty}\left\|x_{n}-T_{r_{i}} x_{n}\right\|=0, \quad \forall i \geq 1
$$


Let $M_{n}=\sup _{i \geq 1}\left\{\left\|x_{n}-T_{r_{i}} x_{n}\right\|\right\}$ for each $n \geq 1$. Then $M_{n} \rightarrow 0$ as $n \rightarrow \infty$. Hence, from (3.33), one has

$$
\begin{aligned}
\left\|x_{n}-z_{n}\right\| & \leq \sum_{i=1}^{n}\left(\theta_{i-1}-\theta_{i}\right)\left\|T_{r_{i}} x_{n}-x_{n}\right\| \\
& \leq \sum_{i=1}^{n}\left(\theta_{i-1}-\theta_{i}\right) M_{n}=\left(1-\theta_{n}\right) M_{n}
\end{aligned}
$$$$
\longrightarrow 0 \text {. }
$$

From (3.26) and (3.34), we obtain

$$
\left\|z_{n}-w_{n}\right\| \leq\left\|z_{n}-x_{n}\right\|+\left\|x_{n}-w_{n}\right\| \longrightarrow 0
$$

Noting that

$$
\begin{aligned}
\sum_{i=1}^{n}\left(\alpha_{i-1}-\alpha_{i}\right)\left(z_{n}-S_{i, n} z_{n}\right) & =\alpha_{n} x_{n}+\left(1-\alpha_{n}\right) z_{n}-w_{n} \\
& =\alpha_{n}\left(x_{n}-w_{n}\right)+\left(1-\alpha_{n}\right)\left(z_{n}-w_{n}\right),
\end{aligned}
$$

we have

$$
\begin{aligned}
& \sum_{i=1}^{n}\left(\alpha_{i-1}-\alpha_{i}\right)\left\langle z_{n}-S_{i, n} z_{n}, z_{n}-p\right\rangle \\
& \quad=\alpha_{n}\left\langle x_{n}-w_{n}, z_{n}-p\right\rangle+\left(1-\alpha_{n}\right)\left\langle z_{n}-w_{n}, z_{n}-p\right\rangle .
\end{aligned}
$$

By Lemma 3.1, we have

$$
\begin{aligned}
\left\|z_{n}-S_{i, n} z_{n}\right\|^{2} & \leq \gamma_{i, n}\left\|z_{n}-p\right\|^{2}+2\left\langle z_{n}-S_{i, n} z_{n}, z_{n}-p\right\rangle \\
& \leq \gamma_{1, n}\left\|z_{n}-p\right\|^{2}+2\left\langle z_{n}-S_{i, n} z_{n}, z_{n}-p\right\rangle .
\end{aligned}
$$

Therefore, combining this inequality with (3.37), we get

$$
\begin{aligned}
\sum_{i=1}^{n}\left(\alpha_{i-1}-\alpha_{i}\right)\left\|z_{n}-S_{i, n} z_{n}\right\|^{2} \\
\leq \gamma_{1, n}\left(1-\alpha_{n}\right)\left\|z_{n}-p\right\|^{2}+2 \alpha_{n}\left\langle x_{n}-w_{n}, z_{n}-p\right\rangle \\
\quad+2\left(1-\alpha_{n}\right)\left\langle z_{n}-w_{n}, z_{n}-p\right\rangle,
\end{aligned}
$$


and hence (noting that $\alpha_{i-1}>\alpha_{i}$ for each $i \geq 1$ )

$$
\begin{aligned}
\left\|z_{n}-S_{i, n} z_{n}\right\|^{2} \leq & \frac{\gamma_{1, n}\left(1-\alpha_{n}\right)}{\alpha_{i-1}-\alpha_{i}}\left\|z_{n}-p\right\|^{2}+\frac{2 \alpha_{n}}{\alpha_{i-1}-\alpha_{i}}\left\langle x_{n}-w_{n}, z_{n}-p\right\rangle \\
& +\frac{2\left(1-\alpha_{n}\right)}{\alpha_{i-1}-\alpha_{i}}\left\langle z_{n}-w_{n}, z_{n}-p\right\rangle .
\end{aligned}
$$

From (3.26), (3.35) and $\lim _{n \rightarrow \infty} \gamma_{1, n}=0$, we have

$$
\lim _{n \rightarrow \infty}\left\|z_{n}-S_{i, n} z_{n}\right\|=0, \quad \forall i \geq 1 .
$$

From the definition of $S_{i, n}$ and (3.41), we have (noting that $\left\{\beta_{i, n}\right\} \subset\left[\kappa_{i}, \mathcal{\kappa}\right) \subset(0,1)$ )

$$
\left\|z_{n}-T_{i}^{n} z_{n}\right\| \leq \frac{1}{1-\beta_{i, n}}\left\|z_{n}-S_{i, n} z_{n}\right\| \longrightarrow 0, \quad \forall i \geq 1 .
$$

We next show (3.42) implies that

$$
\lim _{n \rightarrow \infty}\left\|z_{n}-T_{i} z_{n}\right\|=0, \quad \forall i \geq 1 .
$$

As a matter of fact, from (3.23) and (3.34) we have

$$
\begin{aligned}
\left\|z_{n}-z_{n+1}\right\| & \leq\left\|z_{n}-x_{n}\right\|+\left\|x_{n}-x_{n+1}\right\|+\left\|x_{n+1}-z_{n+1}\right\| \\
& \longrightarrow 0
\end{aligned}
$$

Now, (3.42), (3.44), and Lemma 3.2 imply (3.43).

Since each $T_{i}$ is uniformly continuous and $z_{n} \rightarrow \widehat{x}$ as $n \rightarrow \infty$, one get $\widehat{x} \in F\left(T_{i}\right)$ for each $i \geq 1$ and hence $\widehat{x} \in \bigcap_{i=1}^{\infty} F\left(T_{i}\right)$.

Now we show $\widehat{x} \in \bigcap_{i=1}^{\infty} \operatorname{EP}\left(\Phi_{i}\right)$.

Since every $T_{r_{i}}$ is nonexpansive, from (3.33) and $x_{n} \rightarrow \widehat{x}$, we have $\widehat{x} \in F\left(T_{r_{i}}\right)$ and hence $\hat{x} \in \bigcap_{i=1}^{\infty} F\left(T_{r_{i}}\right)$. Lemma 2.2 shows that $\hat{x} \in \bigcap_{i=1}^{\infty} \mathrm{EP}\left(\Phi_{i}\right)$.

Finally, we prove that $\widehat{x}=P_{\Omega} x$. From $x_{n+1}=P_{D_{n}} x$, one sees

$$
\left\langle x_{n+1}-z, x-x_{n+1}\right\rangle \geq 0, \quad \forall z \in D_{n} .
$$

Since $\Omega \subset D_{n}$ for all $n \geq 1$, one arrives at

$$
\left\langle x_{n+1}-z, x-x_{n+1}\right\rangle \geq 0, \quad \forall z \in \Omega .
$$

Taking the limit for above inequality, we get

$$
\langle\widehat{x}-z, x-\widehat{x}\rangle \geq 0, \quad \forall z \in \Omega .
$$

Hence $\widehat{x}=P_{\Omega} x$. This completes the proof. 
As direct consequences of Theorem 3.3, we can obtain the following corollaries.

Corollary 3.4. Let $C$ be a nonempty closed convex subset of a Hilbert space $H$. Let $\left\{\Phi_{n}\right\}$ be a countable family of bifunctions from: $C \times C$ to $\mathbb{R}$ satisfying (A1)-(A4). Assume that $\Omega=\bigcap_{i=1}^{\infty} \operatorname{EP}\left(\Phi_{i}\right)$ is nonempty and bounded. Let $\left\{r_{n}\right\}$ be a sequence in $(r, \infty)$ with $r>0$. Set $\theta_{0}=1$. The sequence $\left\{x_{n}\right\}$ is generated by $x_{1}=x \in C$ and

$$
\begin{gathered}
z_{n}=\theta_{n} x_{n}+\sum_{i=1}^{n}\left(\theta_{i-1}-\theta_{i}\right) T_{r_{i}} x_{n}, \\
C_{n}=\left\{v \in C:\left\|z_{n}-v\right\| \leq\left\|x_{n}-v\right\|\right\}, \\
D_{n}=\bigcap_{j=1}^{n} C_{j}, \\
x_{n+1}=P_{D_{n}} x, \quad \forall n \geq 1,
\end{gathered}
$$

where $\left\{T_{r_{i}}\right\}$ is defined by (3.8) and $\left\{\theta_{n}\right\}$ is a strictly decreasing sequence in $(0,1)$. Then $\left\{x_{n}\right\}$ converges strongly to $P_{\Omega} x$.

Proof. Putting $T_{i}=I$ for all $i \geq 1$ and $\alpha_{n}=0$ for all $n \geq 1$ in Theorem 3.3, we obtain Corollary 3.4.

Corollary 3.5. Let $C$ be a nonempty closed subset of a Hilbert space $H$. Let $T$ be an asymptotically $\kappa$-strict pseudo-contraction with sequence $\left\{\gamma_{n}\right\} \subset(0, \infty)$ satisfying $\gamma_{n} \rightarrow 0$ as $n \rightarrow \infty$ and $F(T) \neq \emptyset$. Let $\left\{x_{n}\right\}$ and $\left\{u_{n}\right\}$ be sequences generated by $x_{1}=x \in H$ and

$$
\begin{gathered}
z_{n}=\theta_{n} x_{n}+\left(1-\theta_{n}\right) P_{C} x_{n} \\
w_{n}=\alpha_{n} x_{n}+\left(1-\alpha_{n}\right)\left(\beta_{n} I+\left(1-\beta_{n}\right) T^{n}\right) z_{n}, \\
C_{n}=\left\{v \in C:\left\|w_{n}-v\right\| \leq\left\|x_{n}-v\right\|\right\} \\
D_{n}=\bigcap_{j=1}^{n} C_{j}, \\
x_{n+1}=P_{D_{n}} x, \quad \forall n \geq 1
\end{gathered}
$$

where $\left\{\theta_{n}\right\} \subset(0,1),\left\{\alpha_{n}\right\} \subset[0, a]$ with $0<a<1$, and $\left\{\beta_{n}\right\} \subset\left[\kappa, \kappa^{\prime}\right)$ with $\kappa<\mathcal{\kappa}^{\prime}<1$. Then $\left\{x_{n}\right\}$ converges strongly to $P_{F(T)} x$.

Proof. Put $\Phi_{i}(x, y)=0$ for all $x, y \in C$ and set $r_{n}=1$ for all $n \geq 1$ in Theorem 3.3. By Lemma 2.2, we have $T_{r_{i}} x_{n}=P_{C} x_{n}$ for each $i \geq 1$. Hence, by Theorem 3.3, we obtain Corollary 3.5.

Remark 3.6. Our algorithms are of interest because the sequence $\left\{x_{n}\right\}$ in Theorem 3.3 is very different from the known manner. The proof is simple and different from those of others. The main results extend and improve those of Kim and Xu [3], Tada and Takahashi [8], and many others. 
Remark 3.7. Put $\alpha_{0}=1, \theta_{0}=1, \kappa=3 / 4, r=1, \gamma_{i, n}=1 / 4^{i n}, \kappa_{i}=1 / 4+1 /(3+i), \alpha_{n}=1 /(1+n)$, $\theta_{n}=1 / 4+1 / 8 n, \beta_{i, n}=1 / 4+1 /(3+i)+1 / 8 n$ for all $i \geq 1$ and all $n \geq 1, r_{0}=1$, and $r_{n}=1+1 / n$. Then these control sequences satisfy all the conditions of Theorem 3.3.

\section{Acknowledgments}

The authors thank the referees for useful comments and suggestions. This study was supported by research funds from Dong-A University.

\section{References}

[1] K. Goebel and W. A. Kirk, "A fixed point theorem for asymptotically nonexpansive mappings," Proceedings of the American Mathematical Society, vol. 35, pp. 171-174, 1972.

[2] F. E. Browder and W. V. Petryshyn, "Construction of fixed points of nonlinear mappings in Hilbert space," Journal of Mathematical Analysis and Applications, vol. 20, pp. 197-228, 1967.

[3] T.-H. Kim and H.-K. Xu, "Convergence of the modified Mann's iteration method for asymptotically strict pseudo-contractions," Nonlinear Analysis: Theory, Methods \& Applications, vol. 68, no. 9, pp. 28282836, 2008.

[4] Q. Liu, "Convergence theorems of the sequence of iterates for asymptotically demicontractive and hemicontractive mappings," Nonlinear Analysis: Theory, Methods E Applications, vol. 26, no. 11, pp. 1835-1842, 1996.

[5] S. Takahashi and W. Takahashi, "Viscosity approximation methods for equilibrium problems and fixed point problems in Hilbert spaces," Journal of Mathematical Analysis and Applications, vol. 331, no. 1 , pp. 506-515, 2007.

[6] P. L. Combettes and S. A. Hirstoaga, "Equilibrium programming in Hilbert spaces," Journal of Nonlinear and Convex Analysis, vol. 6, no. 1, pp. 117-136, 2005.

[7] R. Wittmann, "Approximation of fixed points of nonexpansive mappings," Archiv der Mathematik, vol. 58, no. 5, pp. 486-491, 1992.

[8] A. Tada and W. Takahashi, "Weak and strong convergence theorems for a nonexpansive mapping and an equilibrium problem," Journal of Optimization Theory and Applications, vol. 133, no. 3, pp. 359-370, 2007.

[9] L. C. Ceng, S. Schaible, and J. C. Yao, "Implicit iteration scheme with perturbed mapping for equilibrium problems and fixed point problems of finitely many nonexpansive mappings," Journal of Optimization Theory and Applications, vol. 139, no. 2, pp. 403-418, 2008.

[10] L. C. Ceng, A. Petruşel, and J. C. Yao, "Iterative approaches to solving equilibrium problems and fixed point problems of infinitely many nonexpansive mappings," Journal of Optimization Theory and Applications, vol. 143, no. 1, pp. 37-58, 2009.

[11] S.-S. Chang, Y. J. Cho, and J. K. Kim, “Approximation methods of solutions for equilibrium problem in Hilbert spaces," Dynamic Systems and Applications, vol. 17, no. 3-4, pp. 503-513, 2008.

[12] Y. J. Cho, X. Qin, and J. I. Kang, "Convergence theorems based on hybrid methods for generalized equilibrium problems and fixed point problems," Nonlinear Analysis: Theory, Methods E Applications, vol. 71, no. 9, pp. 4203-4214, 2009.

[13] X. Qin, Y. J. Cho, and S. M. Kang, "Convergence theorems of common elements for equilibrium problems and fixed point problems in Banach spaces," Journal of Computational and Applied Mathematics, vol. 225, no. 1, pp. 20-30, 2009.

[14] E. Blum and W. Oettli, "From optimization and variational inequalities to equilibrium problems," The Mathematics Student, vol. 63, no. 1-4, pp. 123-145, 1994.

[15] D. R. Sahu, H.-K. Xu, and J.-C. Yao, "Asymptotically strict pseudocontractive mappings in the intermediate sense," Nonlinear Analysis: Theory, Methods \& Applications, vol. 70, no. 10, pp. 3502-3511, 2009. 